This item was submitted to Loughborough's Research Repository by the author.

Items in Figshare are protected by copyright, with all rights reserved, unless otherwise indicated.

\title{
Delayed feedback as a means of control of noise-induced motion
}

PLEASE CITE THE PUBLISHED VERSION

LICENCE

CC BY-NC-ND 4.0

\section{REPOSITORY RECORD}

Janson, Natalia B., Alexander G. Balanov, and E. Scholl. 2019. "Delayed Feedback as a Means of Control of Noise-induced Motion". figshare. https://hdl.handle.net/2134/244. 


\title{
Delayed feedback as a means of control of noise-induced motion
}

\author{
N.B. Janson ${ }^{1,2}$, A.G. Balanov ${ }^{1}$, E. Schöll ${ }^{1}$ \\ ${ }^{1}$ Institut für Theoretische Physik, Technische Universität Berlin, Hardenbergstraße 36, D-10623 Berlin, Germany \\ ${ }^{2}$ Department of Mathematical Sciences, Loughborough University, Loughborough, Leicestershire LE11 $3 T$, UK
}

(Dated: June 22, 2004)

\begin{abstract}
Time-delayed feedback is exploited for controlling noise-induced motion in coherence resonance oscillators. Namely, under the proper choice of time delay, one can either increase or decrease the regularity of motion. It is shown that in an excitable system, delayed feedback can stabilize the frequency of oscillations against variation of noise strength. Also, for fixed noise intensity, the phenomenon of entrainment of the basic oscillation period by the delayed feedback occurs. This allows one to steer the timescales of noise-induced motion by changing the time delay.
\end{abstract}

PACS numbers: 05.40.Ca Noise, 05.45.Gg Control of chaos, applications of chaos

Very often in practical application the need arises to control the properties of oscillations. Usually control assumes an enhancement in regularity of motion [1-3]. However, in some cases, for instance in medical applications, one aims to disorder oscillations, since too strong coherence might be undesirable and even lead to damaging consequences, e.g. epilepsy or Parkinson's disease, $[4,5]$. During the last decade new methods for control of irregular self-sustained oscillations in deterministic systems have been developed, including suppression of chaos by an external (periodic) signal [6], stabilization of unstable periodic orbits embedded in a chaotic attractor by time-discrete control [7], or the use of a time-delayed feedback loop $[8,9]$ for the same purpose.

Whereas the existing methods are designed to control deterministic oscillations or, most recently, noise-induced enhancement of deterministic oscillations [10, 11] and self-oscillations affected by noise [12], there is a large class of systems that do not oscillate autonomously; but if they are forced even by a purely random process featuring no specific timescales, they demonstrate motion resembling a self-oscillatory process $[13,14]$. Prominent representatives of this class are excitable systems like neurons [15], chemical reaction systems [16], and semiconductor nanostructures [17]. The degree of closeness of their oscillations to ideally periodic ones, i.e. coherence, can depend resonantly on noise intensity [13], which is why it was called coherence resonance (CR) [18]. CR has been shown to occur in systems close to bifurcations [19], in excitable systems [18], and in bistable systems [20]. Remarkably, CR oscillators possess the fundamental property of selfoscillators, namely, the ability to synchronize [21].

Frequently the timescale of oscillations in a CR system varies substantially depending on noise intensity. Since the latter is not easily controllable in practice, there is need to make a CR device robust against variation in the properties of noise. Another important task is to find a reliable way to deliberately change the timescales of noise-induced oscillations in a universal way without affecting intrinsic system parameters. Finally, the obvious need is to control the regularity of noise-induced motion. At present, all three problems remain a challenge. In the present Letter we propose to exploit time-delayed feed- back control to tackle all three issues.

As a first example of a CR oscillator, we consider the noisy Van der Pol system closely before the Hopf bifurcation, extended by a delayed feedback loop

$$
\begin{aligned}
& \frac{d x}{d t}=y, \\
& \frac{d y}{d t}=\left(\nu-x^{2}\right) y-\omega_{0}^{2} x+K\left(y_{\tau}-y\right)+D \xi(t) .
\end{aligned}
$$

Here, $x$ and $y$ denote phase variables at time $t$, while $y_{\tau}$ denotes the delayed variable $y(t-\tau) ; K$ is the strength of delayed feedback. $\xi(t)$ is a random variable with Gaussian distribution, zero mean and unity variance, $D$ is the noise intensity. We set the parameters $\nu=-0.01$ and $\omega_{0}=1$ at which a stable focus exists.

First consider $K=0$, i.e. no delayed feedback in Eq. (1). While no limit cycle occurs at $D=0$, application of noise induces oscillatory motion as illustrated by the phase portrait in Fig. 1 (a). The coherence of oscillations may be quantified by the correlation time $t_{c o r}$, estimated from the normalized autocorrelation function $\Psi(s)$ of $y$ as $t_{\text {cor }}=\int_{0}^{\infty}|\Psi(s)| d s$. In Fig. 2(a) the grey (green on-line) line shows $t_{\text {cor }}$ vs $D$ for $K=0$. At small noise intensity $\mathrm{D}$ the oscillations are more coherent.

In deterministic self-oscillatory systems, application of a delayed feedback in the form above acts as follows. If there exists an unstable periodic orbit of period $T$ in the phase space, a feedback with delay time $\tau=T$ can stabilize this orbit in some range of the control strength $K$. A CR system may have no periodic orbits, but noise may induce oscillations with a well-defined timescale that is associated with the spectral peak. We suppose that application of a delayed feedback can act by analogy with a system containing a periodic orbit, provided that $\tau$ is equal or close enough to the basic period $T_{0}$ of the noiseinduced motion [22] without feedback. Namely, it should suppress deviations from a reference state and thus enhance the regularity of oscillations.

To test this expectation, we switch on the control force in Eq. (1). We set $\tau=T_{0}$ with $T_{0}=6.17283951 \approx$ $2 \pi / \omega_{0}$. The phase portrait with control at $K=0.2$ is shown in Fig. 1(b) for the same $D$ as in (a). Fig. 1(b) 
(a)

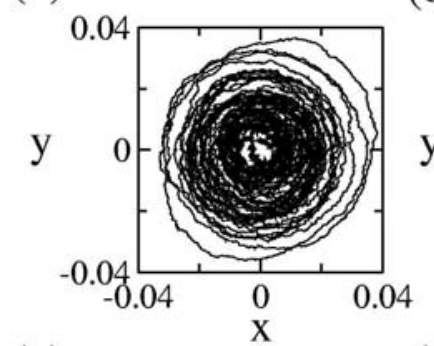

(c)

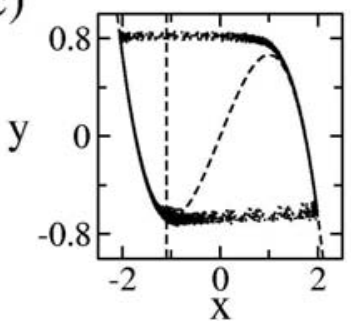

(b)

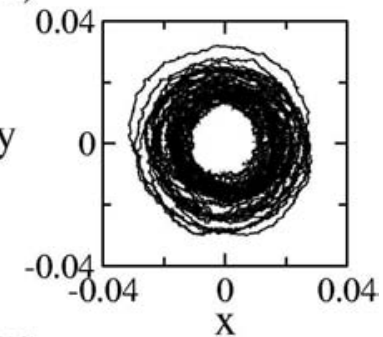

(d)

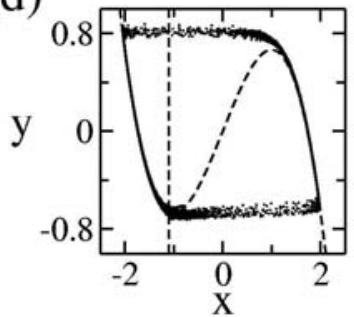

FIG. 1: Phase portraits of noise-induced motion: (a),(b) Van der Pol oscillator at $D=0.003$, (c), (d) FitzHugh-Nagumo system at $D=0.09$ (the dashed lines denote the null-isoclines), (a),(c) $K=0$; (b),(d) $K=0.2, \tau=T_{0}$.

(a)

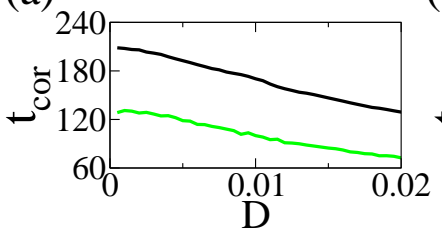

(b)

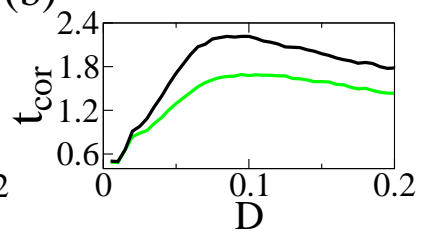

FIG. 2: (color on-line) Correlation time $t_{c o r}$ vs. noise intensity $D$ for (a) Van der Pol oscillator, (b) FitzHugh-Nagumo system. Grey (green on-line) lines: $K=0$, black lines: $K=0.2, \tau=T_{0}$. (b) grey (green on-line) circles: $T_{0}$ for $K=0$, black circles: $T_{1}$ for $K=0.2, \tau=T_{0}$.

reveals a remarkable ordering of the oscillation as compared with Fig. 1(a).

To quantify the ordering due to the feedback, we estimate $t_{c o r}$ in dependence on $D$ as above. It is given for $K=0.2$ by the black line in Fig. 2(a). One can see that for any $D$ the coherence of noise-induced oscillations becomes larger when the delayed feedback loop is switched on and $\tau$ is close to $T_{0}$. On the other hand, it was found that if $\tau$ is far from an integer multiple of $T_{0}$, the coherence of oscillations, on the contrary, decreases. Also, for $\tau=T_{0}$ the coherence of noise-induced oscillations in Eq. (1) was found to increase monotonically with increasing $K$. In the following we fix $K=0.2$. Next, we study how the feedback can affect the system's timescales. For this purpose, we set the noise at the intensity $D=0.003$ as for Figs. 1(a), (b), and follow the evolution of Fourier power spectra with $\tau$, which is illustrated by Fig. 3(a). Without feedback $(\tau=0)$, system (1) has one pronounced peak $f_{0}$ in the spectrum. As $\tau$ increases from zero, the peak frequency, height and width change. At $\tau>8$, new peaks that change their positions, heights and widths with $\tau$ become clearly visible.

Since the control parameter of delayed feedback is the time interval $\tau$, we propose to describe the response of the system in terms of periods rather than frequencies. With feedback, we select all spectral peaks, and for each peak introduce the period $T$ as the inverse of the peak frequency. Denote the period of highest peak by $T_{1}$. The dependences of $T$ on $\tau$ are given in Fig. 4 (a): $T_{1}$ by circles (yellow on-line), other $T$ by crosses (blue on-line). One can see that variation of $\tau$ changes $T_{1}$ in a certain range, doing so most effectively as $\tau<T_{0} / 2$. As $\tau$ increases beyond $T_{0} / 2, T_{1}$ drops quickly, and again increases with $\tau$ with a similar slope as before. After $\tau$ has increased by about $T_{0}, T_{1}$ again drops abruptly to a lower branch, and again follows $\tau$, although with a smaller slope. These abrupt transitions to successive lower branches occur roughly every $T_{0}$ time units, and each subsequent entrainment happens at a lower slope. The plot of $T_{1}$ vs $\tau$ exhibits a piecewise approximately linear dependence, the larger the $\tau$, the closer each segment is to a straight line.

The numerical results obtained above for the Van der Pol system can be understood in terms of a general theory of a canonical nonlinear oscillator with time-delayed feedback

$$
\ddot{x}+f(x, \dot{x})-K\left(\dot{x}_{\tau}-\dot{x}\right)=0 .
$$

Note that Eq. (1) fits the form Eq. (2) if rewritten as a single second-order differential equation with $f(x, \dot{x})=$ $-\left(\nu-x^{2}\right) \dot{x}+\omega_{0}^{2} x$ and $y=\dot{x}$. Without feedback $(K=0$ or $\tau=0)$ the fixed point $\left(x_{0}, 0\right)$ is a stabe focus if

$$
0<\frac{\partial f}{\partial \dot{x}}<2 \sqrt{\frac{\partial f}{\partial x}}
$$

where partial derivatives are taken at the fixed point. In Eq. (1) $x_{0}=0$, and Eq. (3) is true for the given parameters. Setting $K>0$ does not change $x_{0}$. Either with, or without feedback the noise-induced oscillations take place in the close vicinity of the fixed point. It is to be expected that the motion is influenced by the local properties of this point. At $\tau=0$ the stable focus has a pair of complex conjugate eigenvalues $\lambda_{0}=p_{0} \pm i q_{0}, p_{0}<0$, $q_{0} \neq 0$, and the value of $q_{0}$ should give an estimate of the angular frequency. Indeed, the only peak of the power spectrum (Fig. 3(a)) has frequency $f_{0} \approx\left|q_{0}\right| / 2 \pi$. With $\tau>0$, the system becomes infinite-dimensional, and possesses a countable set of eigenvalues $\lambda$. In order to exclude that the delayed feedback might induce the birth of a stable limit cycle via a Hopf bifurcation, thus providing a trivial explanation for the remarkable ordering of oscillations, we perform a linear stability analysis of the fixed point of Eq. (2). Following the standard routine of linearizing Eq. (2) around the fixed point [23], the 
characteristic equation for $\lambda$ is derived:

$$
\lambda^{2}+\lambda \frac{\partial f}{\partial \dot{x}}+\frac{\partial f}{\partial x}-K \lambda\left(e^{-\lambda \tau}-1\right)=0,
$$

Substituting $\lambda=p+i q$, real and imaginary parts can be separated. The condition for a Hopf bifurcation is $p=0$, $q \neq 0$. Substituting it into the imaginary part of Eq. (4) we obtain:

$$
\cos q \tau=\frac{K+\partial f / \partial \dot{x}}{K} .
$$

Since the right-hand side is larger than unity due to Eq. (3), the Hopf bifurcation condition is not satisfied for any $K$ and $\tau$. Thus, the delayed feedback in the given form cannot induce a Hopf bifurcation.

The numerical solution of Eq. (4) with $f(x, \dot{x})$ from Eq. (1) yields the eigenvalue spectrum $\lambda=p+i q$ as a function of $\tau$. The eigenperiods defined as $T^{e}=2 \pi /|q|$ (dots in Fig. 4(a)) coincide remarkably with the inverse peak frequencies of the power spectrum of the noiseinduced oscillations as a function of $\tau$. The corresponding real parts $p$ are given by dots in Fig. 4(b) (the seven largest $p$ are shown). All $p$ remain negative, but, as seen from Fig. 4(b), nonmonotonically change with $\tau$. As $\tau$ increases, separate branches of $p$ cross, thus providing a striking explanation of the strongly nonmonotonic, discontinuous evolution of the dominant spectral peak of the noise-induced motion under delayed feedback: The period $T_{1}$ of the highest spectral peak (circles (yellow on-line) in Fig. 4(a)) always coincides with the period $T^{e}$ of the least stable eigenmode, i.e. the one with the largest real part which we denote as $p_{1}$ (circles (yellow on-line) in Fig. 4(b)). The more stable eigenmodes result in the side peaks of the frequency spectrum. The more stable the modes are, the lower the peaks are.

As $p_{1}$ oscillates with $\tau$, the degree of stability of the fixed point of the deterministic system is modulated, thus leading to modulation of the coherence of the stochastic motion, quantified by the correlation time $t_{c o r}$ (solid line (green on-line) in the upper part of Fig. 4(b)). The local maxima of coherence occur when $p_{1}$ is close to zero, and $T_{1}$ is close to $T_{0}$.

The entrainment of $T_{1}$ by $\tau$, which manifests itself in the almost piecewise linear dependence of $T_{1}$ on $\tau$ for large $\tau$, can be understood as follows. As shown above, it is related to the eigenvalue whose real part $p_{1}$ is closest to zero. Assuming $p_{1} \approx 0$ in Eq. (4) we obtain Eq. (5). With $\partial f / \partial \dot{x}=-\nu \ll K$, and $(K-\nu) / K \approx 1$, this gives $\cos (q \tau) \approx 1$ and $|q| \tau \approx 2 \pi n$, where $n$ is integer. Then the eigenperiod $T^{e}$ is

$$
T^{e}=2 \pi /|q| \approx \frac{\tau}{n}
$$

As illustrated by Fig. 4(b), $p_{1}$ is close enough to zero only for large $\tau$, for which the relation (6) holds most accurately. To obtain the location of the maxima of $p_{1}$, i.e. the maxima of coherence, substitute $p_{1} \approx 0, q=$
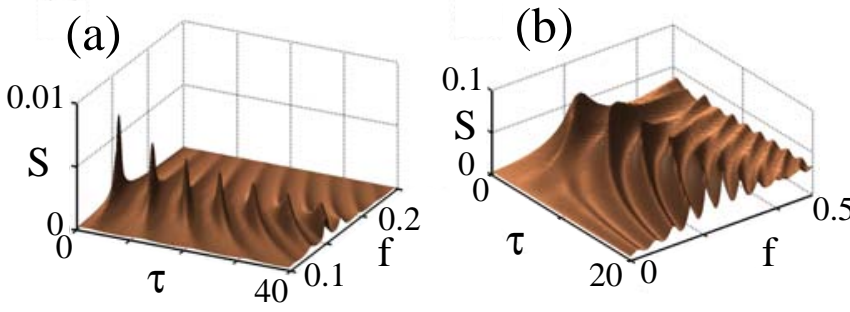

FIG. 3: Fourier power spectra of noise-induced oscillations in dependence on $\tau$ for (a) Van der Pol oscillator at $D=0.003$; (b) FitzHugh-Nagumo system at $D=0.09, K=0.2$. The spectrum is computed from $y$. (a)
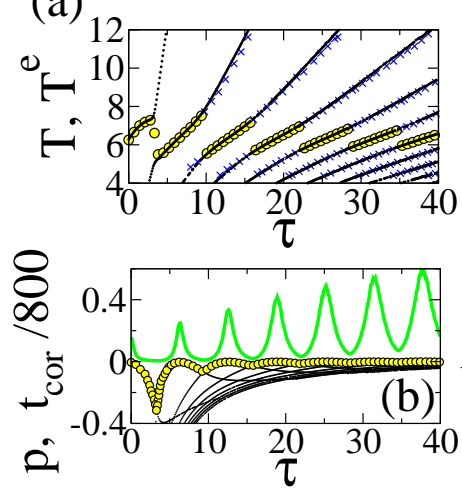

(c)
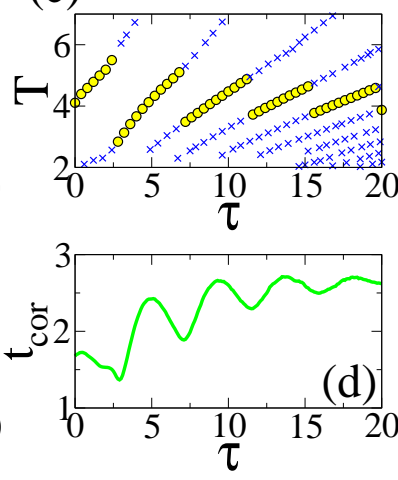

FIG. 4: (color on-line) Spectral peaks, coherence and eigenvalues vs $\tau$ at $K=0.2$. (a),(b): Van der Pol oscillator at $D=0.003,(\mathrm{c}),(\mathrm{d})$ : FitzHugh-Nagumo system at $D=0.09$. (a),(c) crosses (blue on-line): $T$, circles (yellow on-line): $T_{1}$, black dots: $T^{e}$. (b),(d) solid line (green on-line): $t_{c o r},(\mathrm{~b})$ black dots: seven largest $p$, circles (yellow on-line): $p_{1}$.

$2 \pi n / \tau$ into the real part of Eq. (4), which yields $\tau=$ $2 \pi n / \omega_{0}=n T_{0}$.

To summarize, delayed feedback applied to an oscillatory system of the form (2), gives rise to a countable set of eigenmodes of the fixed point, whose eigenperiods and stability are controlled by $\tau$. The highest peak in the spectrum of the noise-induced motion is due to excitation of the least stable eigenmode. The coherence of oscillations is the higher, the less stable the mode is. The range of modulation of the peak frequency is largest for small tau and large $\mathrm{K}$.

Next, we consider another example of a CR oscillator, the FitzHugh-Nagumo system, which serves as a prototype of an excitable system. Extending it again by a delayed feedback loop, we obtain

$$
\begin{aligned}
\epsilon \frac{d x}{d t} & =x-\frac{x^{3}}{3}-y \\
\frac{d y}{d t} & =x+a+K\left(y_{\tau}-y\right)+D \xi(t) .
\end{aligned}
$$

We set the parameters $\epsilon=0.01$ and $a=1.1$ such that 
a stable node is the only attractor of the system in the absence of feedback.

Without feedback $(K=0)$, the mechanism for inducing oscillations by noise is different from the Van der Pol oscillator (1). In Fig. 1(c), (d) dashed lines show the null-clines defined by $d y / d t=0$ (vertical) and by $d x / d t=0$ (cubic parabola). They intersect at the fixed point, which is slightly displaced to the left of the minimum of the parabola for the parameters chosen. The null-clines divide the phase plane into four regions with different directions of phase velocity. Dots in Fig. 1(c) show the phase portrait with noise $D=0.09$.

In Fig. 2(b) the grey (green on-line) line shows $t_{c o r}$ for Eq. (7) versus noise intensity, exhibiting a distinct maximum at $D=0.09$. Also, grey (green on-line) circles show the basic period $T_{0}$ of oscillations. Unlike Eq. (1), here $T_{0}$ changes substantially with noise, as was earlier shown in [20].

Now, switch on the feedback with $K=0.2$ and set $\tau$ equal to the value of $T_{0}=4.12694$ at optimum noise. The black line in Fig. 2(b) denotes $t_{c o r}$ vs $D$, and shows that for any $D$ the coherence of oscillations is higher with the feedback. However, this feature is not visible in the phase portrait (Fig. 1(d)). Black circles in Fig. 2(b) show the basic period $T_{1}$ with feedback. It is evident that delayed feedback substantially reduces the variation of the noise-induced basic timescale. Note, however, that this may not be so if $\tau$ is very different from $T_{0}$.

Next, we study how the feedback can control the timescales and the regularity of noise-induced motion. Fix $D$ at an optimum value $0.09, K$ at 0.2 and change $\tau$. The spectrum in dependence on $\tau$ is given in Fig. 3(b). With increasing $\tau$, the spectral peaks move towards zero, and the spectrum is gradually enriched by new peaks. As with the Van der Pol oscillator, select all visible peaks with periods $T$, and denote the period of the highest peak as $T_{1}$. In Fig. 4(c) $T$ of several peaks are given by crosses (blue on-line), and $T_{1}$ by circles (yellow on-line), depending on $\tau$. These dependencies are qualitatively very much like those for Eq. (1) in Fig. 4(a). In Fig. $4(\mathrm{~d}) t_{\text {cor }}$ is given depending on $\tau$, exhibiting oscillatory features. Local maxima of coherence occur when $T_{1}$ is equal to $T_{0}$. Unlike in case of the Van der Pol oscillator, the mechanism of delayed feedback control can not be explained by a local analysis of the fixed point since the oscillations are characterized by large excursions in phase space. Rather, a global analysis would be needed which is clearly beyond the scope of the present Letter.

In conclusion, time-delayed feedback in the form of the difference between the current and a delayed state of the system can be used to control oscillations that are induced merely by noise. The most crucial parameter of such a control is the time delay $\tau$, depending on which the coherence of noise-induced oscillations increases or decreases. With this, a phenomenon of entrainment of the basic period of noise-induced motion by the time delayed feedback is discovered. The latter ability is somehow reminiscent of classical synchronization phenomena, in that the externally imposed timescale $\tau$ tunes the basic period of oscillations in the system, although it involves quite different mechanisms.

This work was supported by DFG in the framework of $\mathrm{Sfb} 555$. The authors gratefully acknowledge discussions with A. Nikitin and A. Amann.
[1] D.J. Christini, K.M. Stein, S.M. Markowitz, S. Mittal, D.J. Slotwiner, M.A. Scheiner, S. Iwai, B.B. Lerman, PNAS 98, 5827 (2001).

[2] N. Baba, A. Amann, E. Schöll, and W. Just, Phys. Rev. Lett. 89, 074101 (2002).

[3] A. G. Balanov, N. B. Janson, P. V. E. McClintock, R. W. Tucker and C.H.T. Wang, Chaos, Solitons \& Fractals 15, 381 (2003).

[4] F. E. Dreifuss et al, Epilepsia 22, 489 (1981).

[5] P. Tass, Phys. Rev. E 66, 036226 (2002).

[6] D.E. Postnov, A.G. Balanov, O.V. Sosnovtseva, E. Mosekilde, Phys. Lett. A 283, 195 (2001).

[7] E. Ott, C. Grebogi, and J. Yorke, Phys. Rev. Lett. 64, 1196 (1990).

[8] K. Pyragas, Phys. Lett. A 170421 (1992).

[9] J. E. S. Socolar, D. W. Sukow, and D. J. Gauthier, Phys. Rev. E 50, 3245 (1994).

[10] L. Gammaitoni, M. Locher, A. Bulsara, P. Hanggi, J. Neff, K. Wiesenfeld, W. Ditto, M.E. Inchiosa, Phys. Rev. Lett. 82, 4574 (1999).

[11] J.F. Lindner, J. Mason, J. Neff, B.J. Breen, W.L. Ditto, A.R. Bulsara, Phys. Rev. E 63, 041107 (2001).

[12] D. Goldobin, M. Rosenblum, and A. Pikovsky, Phys. Rev. E 67, 061119 (2003).
[13] H. Gang, T. Ditzinger, C.Z. Ning, H. Haken, Phys. Rev. Lett. 71, 807 (1993).

[14] W.-J. Rappel and S.H. Strogatz, Phys. Rev. E 50, 3249 (1994).

[15] S.-G. Lee, A. Neiman, and S. Kim, Phys. Rev. E 57, 3292-3297 (1998).

[16] K. Miyakawa and H. Isikawa, Phys. Rev. E 66, 046204 (2002).

[17] J. Unkelbach, A. Amann, W. Just, and E. Schöll, Phys. Rev. E 68, 026204 (2003).

[18] A. Pikovsky and J. Kurths, Phys. Rev. Lett. 78, 775 (1997).

[19] A. Neiman, P.I. Saparin and L. Stone, Phys. Rev. E 56, 270 (1997).

[20] B. Lindner and L. Schimansky-Geier, Phys. Rev. E 61, 6103 (2000).

[21] S.K. Han, T.G. Yim, D.E. Postnov and O.V. Sosnovtseva, Phys. Rev. Lett. 83, 1771 (1999).

[22] By basic period we mean the inverse of the frequency of the highest spectral peak.

[23] J.K. Hale, Functional differential equations (Applied mathematical sciences 03, Springer), 1971. 